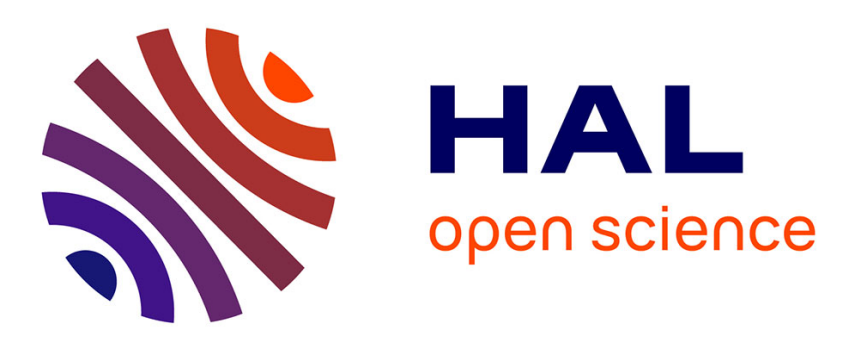

\title{
L'influence des Églises évangéliques sur le soft power américain en Égypte
}

Dominique Cadinot

\section{To cite this version:}

Dominique Cadinot. L'influence des Églises évangéliques sur le soft power américain en Égypte. Amerika - Mémoires, identités, territoires, 2015, 10.4000/amerika.5897 . halshs-01421647

\section{HAL Id: halshs-01421647 https://shs.hal.science/halshs-01421647}

Submitted on 3 Jan 2017

HAL is a multi-disciplinary open access archive for the deposit and dissemination of scientific research documents, whether they are published or not. The documents may come from teaching and research institutions in France or abroad, or from public or private research centers.
L'archive ouverte pluridisciplinaire $\mathbf{H A L}$, est destinée au dépôt et à la diffusion de documents scientifiques de niveau recherche, publiés ou non, émanant des établissements d'enseignement et de recherche français ou étrangers, des laboratoires publics ou privés. 


\section{Amerila Amerika \\ Mémoires, identités, territoires}

$12 \mid 2015$

La mort : imaginaires et sociétés

\section{L'influence des Églises évangéliques sur le soft power américain en Égypte}

\section{Dominique Cadinot}

revues.org

Édition électronique

URL : http://amerika.revues.org/5897

ISSN : 2107-0806

\section{Éditeur}

LIRA-Université de Rennes 2

Ce document vous est offert par Aix Marseille Université

\section{Aix $*$ Marseille}

Référence électronique

Dominique Cadinot, "L'influence des Églises évangéliques sur le soft power américain en Égypte », Amerika [En ligne], 12 | 2015, mis en ligne le 01 juillet 2015, consulté le 22 décembre 2016. URL : http:// amerika.revues.org/5897; DOI : 10.4000/amerika.5897

Ce document a été généré automatiquement le 22 décembre 2016.

(c) Tous droits réservés 


\title{
L'influence des Églises évangéliques sur le soft power américain en Égypte
}

\author{
Dominique Cadinot
}

\section{La liberté religieuse est-il exportable? Analyse de stratégies américaines en Égypte (2000-2013)}

1 La typologie des cultures politiques américaines dressée par l'historien Walter Russell Mead, développe deux écoles de pensée attachées à deux figures mythiques qui caractérisent particulièrement les orientations de la politique étrangère américaine dans la dernière décennie du $\mathrm{XX}^{\mathrm{e}}$ siècle $:$ le courant hamiltonien qui rassemble les partisans de la puissance économique, et le courant wilsonien visant à diffuser les valeurs de démocratie et de liberté à travers le monde. Toutefois, l'homogénéité parfaite ne pouvant être historiquement constatée, la présidence du démocrate Bill Clinton, entre 1993 et 2001, aurait été, d'après l'historien, le théâtre d'une rivalité permanente ; la construction d'un marché économique global et la promotion des valeurs de l'Amérique formant alternativement la clef de voûte de la politique étrangère états-unienne de l'époque (175). Alors qu'il effectue un voyage en Chine, Bill Clinton endosse l'habit wilsonien et tient le discours suivant :

J'ai la conviction, comme le peuple américain, que la liberté religieuse, la liberté de parole ou de s'assembler sont des droits communs à tous les peuples [...]. C'est pour promouvoir ces droits que nos Pères fondateurs, dans notre Déclaration d'Indépendance, ont engagé nos vies, nos fortunes et notre honneur sacré. ${ }^{1}$ (cité dans Auerswald, 180)

Parce qu'elle forme depuis toujours la pierre angulaire de l'édifice national, la liberté religieuse inscrite dans le premier amendement à la Constitution tient naturellement une place de choix dans le propos du président. Toutefois, la ratification, par Bill Clinton en 1998, de l'International Religious Freedom Act (IRFA) constitue une étape significative car, 
pour la première fois, cette loi inscrit officiellement la promotion de la liberté religieuse dans l'histoire de la diplomatie américaine. Il convient de rappeler que faisant suite à la « révolution républicaine » de 1994, les deux chambres du Congrès sont alors aux mains des néo-conservateurs que soutiennent les Églises évangéliques et fondamentalistes, comme la puissante Southern Baptist Convention. Emanant donc en fait de la droite chrétienne ${ }^{2}$ qui imprime sa marque aux prérogatives du Congrès, ce nouveau texte de loi autorise la création d'une agence fédérale (USCIRF - United States Commission on International Religion Freedom) chargée de remettre chaque année aux législateurs un rapport sur les conditions de la liberté de culte dans le monde.

3 Si la constitutionalité d'initiatives visant à impliquer le gouvernement fédéral dans les affaires religieuses d'autres pays est une question légitime, cet article propose une analyse de la mise en exergue du référent religieux dans la conduite des affaires étrangères. Plus précisément, il s'agira d'abord, à partir d'une lecture critique des rapports de l'USCIRF publiés entre 2000 et 2013, d'identifier les implications idéologiques de l'engagement de la droite religieuse et de ses alliés fondamentalistes en faveur des minorités confessionnelles à l'étranger. Dans un second temps, l'article propose de confronter ce discours à la réalité sociale telle qu'elle est perçue et analysée par les opposants autochtones aux initiatives américaines. En effet, dans plusieurs pays, de nombreux citoyens se sont opposés vigoureusement à toute ingérence des États-Unis. Les chrétiens d'Orient formant depuis toujours la cible privilégiée des "missionnaires" américains, nous nous intéresserons en particulier aux réactions des intellectuels et des responsables politiques en Égypte ${ }^{3}$. Comment en effet comprendre les réticences de certains dignitaires égyptiens au travail des Américains pour le respect de la liberté religieuse universelle, surtout lorsque l'on est copte ou chiite et que l'on subit de manière récurrente la violence de ses concitoyens affiliés à un autre groupe confessionnel? Il s'agira, au bout du compte, de mettre au jour les effets de "l'inévitable responsabilité » américaine sur les rapports de force entre les communautés qui forment le corps social égyptien ${ }^{4}$.

\section{Incrimination et implicite : lecture critique des rapports de I'USCIRF}

4 Bien que présentes sur la scène politique depuis le premier mandat de Ronald Reagan, les Églises évangéliques profitent en 1998 du vide laissé dans les couloirs du pouvoir par les libéraux (au sens américain du terme) pour appeler de leurs vœux l'instauration d'une politique fédérale destinée à promouvoir la liberté religieuse à l'étranger. Cette mobilisation dont l'une des expressions est la participation à la rédaction annuelle de rapports de l'USCIRF offre ainsi à la mouvance chrétienne l'occasion exceptionnelle de promouvoir dans des documents officiels sa propre vision du monde.

5

Chaque année, les pays sous observation sont classés dans deux listes : la Watch List qui regroupe, comme son nom l'indique, les pays à surveiller, et la liste des CPC ou Countries of Particular Concern qui épingle les pays fautifs. Si l'Iran, le Pakistan ou la Chine figurent invariablement dans chaque rapport, l'Égypte, apparaît dans la Watch List en 2002, avant d'être nommée dans la seconde à partir de 2011, lorsque des soulèvements populaires exceptionnels provoquent la chute du régime égyptien. 
6 En premier lieu, on remarque que les rapports de la commission se densifient considérablement, s'enrichissent d'illustrations, mais comportent aussi de nombreux paragraphes qui sont copiés-collés d'une année sur l'autre ${ }^{5}$. Le chapitre consacré au seul bilan des libertés religieuses en Égypte passe ainsi d'une seule page en 2000 à plus de quinze pages en 2013. Les dernières parutions comportent donc une sélection des événements politiques de l'année suivie d'un compte-rendu des persécutions ou discriminations exercées contre les minorités confessionnelles égyptiennes. Les communautés recensées par la commission sont: les bahaïs (religion syncrétique d'origine iranienne), les chiites, les coranistes (un courant de l'islam), la communauté juive, les témoins de Jéhovah. Le plus grand nombre de pages est consacré aux chrétiens d'Orient et aux coptes orthodoxes précisément ${ }^{6}$. Dans le rapport de 2006, par exemple, trois pages traitent de la situation des chrétiens contre une demi-page pour chaque autre minorité. Les observateurs y présentent un inventaire chronologique des évènements de l'année, résumant de manière détaillée les attentats, les meurtres ou les cas de discrimination à l'encontre des Égyptiens de confession chrétienne. Sont rapportés par exemple les incidents violents survenus dans la ville d'Esna en Haute-Égypte, les arrestations arbitraires ou les défaillances des procédures judiciaires contre les responsables de violences confessionnelles (Uscirf, 2006, 223). Cette prioritisation du cas chrétien avait déjà été, il faut le souligner, l'une des raisons qui avaient entravé en 1997 le passage d'un premier projet de loi appelé le Wolf-Specter bill. À l'époque, l'argumentaire évangélique ne s'était pas encore associé à celui des humanistes séculiers. En juin 1997, lors d'une audition préliminaire devant la commission sénatoriale en charge des Affaires étrangères, Nina Shea expliquait que jamais dans l'histoire les chrétiens n'avaient autant souffert et que le discours séculier-libéral de promotion des droits de l'homme avait montré ses limites :

Les chrétiens forment la communauté religieuse la plus persécutée du monde aujourd'hui [...] Voilà quelque chose que l'on ignore. Pourquoi ? Eh bien, M. le Président de la commission, je crois que nos habitudes discriminatoires et notre myopie séculière nous empêchent de reconnaître le problème. (1) ${ }^{7}$

7 À la lecture de ces treize rapports, on remarque donc que, si au gré des successions l'angle d'investigation s'est parfois élargi pour inclure de manière plus équilibrée les autres minorités confessionnelles (voir les rapports de 2010 et 2011), la lutte pour la liberté de culte signifie toujours et avant tout, pour les membres de la commission, la sauvegarde du christianisme. Le fait que chacune des commissions ait compté en son sein deux ou trois représentants du courant évangélique (comme Richard Land de la Southern Baptist Convention), souvent épaulés par un catholique conservateur (comme Charles J. Chaput, le très orthodoxe archevêque de Philadelphie), n'est pas sans lien au soutien apporté aux chrétiens d'Égypte.

8 Si l'on s'intéresse maintenant à l'examen proposé du contexte social égyptien, on relève que très souvent l'angle adopté est celui du fait divers. Les rédacteurs s'appuient en outre sur des exemples de violence qui, expliquent-ils, ont fait suite à de simples rumeurs ou allégations. Ainsi dans le rapport de 2008, par exemple, on peut lire: «En février 2007, des groupes de Musulmans auraient incendié en Haute-Égypte des commerces chrétiens suite à des rumeurs faisant état d'une liaison entre un chrétien et une musulmane » (Uscirf, 2008, 224) ${ }^{8}$. En dehors des cas de vengeances personnelles, de vendettas familiales ou d'agressions sexuelles, rares sont les explications fournies quant à des motivations plus profondes d'ordre politique ou des conditions socio-économiques ayant pu déclencher de tels actes. Le rôle joué par les dignitaires religieux dans la vie des 
communautés n'est par exemple jamais évoqué. Il n'est nulle part fait mention du Pape Chenouda III, patriarche de l'Église copte orthodoxe et interlocuteur privilégié du raïs égyptien de 1971 à 2012. De même, les manœuvres orchestrées par la confrérie des Frères musulmans pour acquérir des électeurs et se hisser au pouvoir après 2011 ne sont pas reliées à la violence confessionnelle. Le même paragraphe synthétique est reproduit à l'identique d'une année sur l'autre et inséré en fin de rapport. En somme, les affrontements interconfessionnels sont souvent relatés comme s'ils résultaient d'un déchaînement de haine irraisonné, et indépendant de toute influence cléricale ou communautaire. La nature et les ressorts de la conflictualité ne sont pas l'objet d'une analyse approfondie. Toutefois, dès 2000, il est rapporté que les tensions entre chrétiens et musulmans ont été aggravées par le pouvoir égyptien qui a organisé de jure la discrimination. Les rédacteurs soulignent en particulier les difficultés rencontrées pour obtenir du gouvernement l'autorisation et les fonds nécessaires à la construction ou restauration des édifices cultuels (Uscirf, 2000, 20). Autre explication contextuelle, mais fournie bien plus tard en 2005, l'état d'urgence imposé par la force pendant plus de trente années (1981-2012) et prétexte à une législation discriminatoire (Uscirf, 2005, 108). Les accusations formulées par les membres de la commission portent donc uniquement sur la fonction régulatrice du pouvoir étatique et laissent généralement de côté la fonction médiatrice de la société civile. En outre, le portrait brossé de la société civile égyptienne entérine le modèle de structuration social autours des communautés religieuses tel qu'il est conçu et exploité par le pouvoir mais, de fait, occulte la dimension politique.

Par conséquent, dans le modèle idéologique dessiné par les Américains, la liberté religieuse de l'individu prime au dépend des initiatives collectives. C'est l'individu, croyant et libre qui est le vecteur d'harmonie sociale. Cette focalisation sur l'individu est, selon Sébastien Fath, un élément essentiel du discours évangélique américain: "Au contraire $\mathrm{du}$ protestantisme mainline, [ce discours] favorise l'individu plus que l'institution, plus que l'infrastructure » (66). Mais surtout, il encourage, selon Mokhtar Ben Barka, « une indifférence aux questions de justice sociale » $(1998,20)$. Le rapport de l'année 2008, en effet, ne fait nulle part mention des mouvements de grève nationale organisée par les travailleurs de l'usine de textile Ghazl El-Mahalla. L'effet implicite de l'action américaine est par conséquent de permettre au croyant égyptien de transcender "les hiérarchies du pouvoir, du savoir ou de la fortune » (Keppel, 61).

Par ailleurs, autre élément significatif, dans tous les rapports de la commission, un chapitre est consacré à la question du prosélytisme religieux. Or, comme chacun sait, l'exercice du prosélytisme revêt une importance capitale pour les évangéliques. M. Ben Barka rappelle que l'expérience individuelle de la conversion (et du baptême) est privilégiée au dépend de la foi héritée; d'où la valeur accordée à la renaissance spirituelle. Être born again implique d'être un exemple, et donc de propager la foi : « Le zèle du convertisseur ne s'arrête pas à tous ceux qui sont près de lui : les frontières du monde en sont les limites. Il faut que la Bonne Nouvelle parvienne à l'humanité entière » $(2008,94)$. Sans jamais y faire clairement référence, la commission évoque les difficultés rencontrées par les missionnaires protestants américains (appelés Protestant foreign workers) qui sous diverses couvertures font œuvre de prosélytisme auprès des populations égyptiennes9. Les prises d'otages en Égypte ou dans d'autres pays du Monde arabe de pasteurs itinérants, comme celle en 2012 de Michel Louis fondateur d'une Église Pentecôtiste à Boston, attestent la présence difficilement quantifiable de ces fantassins de la foi ${ }^{10}$. 
11 A la suite du paragraphe consacré au prosélytisme religieux, tous les rapports dénoncent les discriminations ou les persécutions subies par les Égyptiens musulmans convertis au christianisme. Aucun exemple de violences à l'encontre d'Égyptiens chrétiens convertis à l'islam n'est mentionné. L'analyse proposée des motifs de conversion est là aussi superficielle. Seuls les cas de conversion matrimoniale sont évoqués. Le lecteur apprend donc qu'en matière de divorce, l'Église chrétienne orthodoxe est très stricte puisqu'elle ne valide la procédure qu'en cas d'adultère avéré. C'est pour cette raison que de nombreux coptes se convertissent à l'islam dans un premier temps pour ensuite, le divorce obtenu, se reconvertir au christianisme (on parle donc de re-converts) ${ }^{11}$. Si les rédacteurs restent discrets sur la rigidité des autorités cléricales, ils dénoncent par contre les difficultés rencontrées par les « reconvertis » pour obtenir la mention de leur dernière affiliation religieuse sur leurs passeports ou cartes d'identité. Depuis le début des années 2000, des centaines de chrétiens reconvertis ont en effet engagé des actions en justice pour recouvrer leur identité religieuse originelle. Mais, en dehors de cette perspective utilitariste, aucune analyse n'est offerte sur les motivations autres que matrimoniales ayant amené les convertis à renier leur foi originelle. Même si le processus de conversion demeure un sujet de débats, de nombreuses recherches ont mis au jour l'enchevêtrement des motivations. Comme l'ont démontré, par exemple, les travaux de Raymond F. Paloutzian, J. T. Richardson et L. R. Rambo, la conversion peut constituer une ressource efficace pour renégocier les identités et les statuts: «Se convertir peut entraîner des modifications profondes et déterminantes tant au niveau des objectifs, sentiments, attitudes ou comportements qu'au niveau des traits plus intimes de la personnalité comme la perception de son identité ou le sens donné à sa vie» $(1070)^{12}$. Plus précisément, dans une étude consacrée au phénomène de conversion à l'islam en Égypte, Laure Guirguis observe : « [les chrétiens convertis] voient dans l'islam un moyen [...] de se défaire de la haine de soi instillée au corps individuel copte par la multiplication des signes d'infériorité dans les espaces collectifs » (76). La complexité des enjeux identitaires liés au changement d'affiliation religieuse n'est donc pas prise en compte dans le tableau dressé du paysage confessionnel égyptien où pourtant la foi détermine souvent le statut social.

12 Même si l'architecture principale et le contenu thématique des rapports de l'USCIRF restent inchangés, des variations dans le champ d'investigation sont toutefois repérables. Alors qu'en 2003, les membres de la commission ont pour la plupart des valeurs morales plutôt conservatrices (qu'elles soient catholiques, protestantes ou juives) ${ }^{13}$, l'attention des rédacteurs se porte uniquement sur les persécutions limitant la liberté de culte. L'année suivante, la nomination par la démocrate Nancy Pelosi, speaker à la chambre des Représentants, de deux nouveaux commissaires -Patti Chang, militante féministe de Californie et Khaled Abou El Fadl, juriste spécialiste de droit islamique- coïncide avec un élargissement des libertés sous observation. Ainsi la version de 2003 annonce: "De graves problèmes de discrimination à l'encontre de certains groupes religieux persistent dans de nombreuses régions d'Égypte» (Uscirf, 15). En revanche, celle de 2004 débute ainsi : «De graves problèmes de discrimination ou violations d'autres droits à l'encontre de communautés religieuses persistent dans de nombreuses régions d'Égypte » et continue avec l'observation suivante : «Le bilan de la situation des droits de l'homme en Égypte n'est pas bon car il atteste de pratiques répressives qui enfreignent considérablement la liberté de pensé, de conscience et de religion » (Uscirf, 72) ${ }^{14}$. Cette variation discrète mais porteuse de sens révèle les désaccords qui au sein même de la 
structure d'observation ont opposé par intermittence les membres de la commission. Elle témoigne aussi des convictions politiques ou morales des administrations qui se sont succédées entre 1998 et 2013. Toutefois, en dépit de ces moments de tension, dans les éditions de 2009, 2010 et 2013 le dernier paragraphe porte un titre qui consacre clairement la primauté de la liberté de culte sur toute autre liberté : «Comment protéger la liberté religieuse et autres droits associés » (Uscirf 2013, 29) ${ }^{15}$.

Il devient donc évident, comme l'a aussi souligné Dominique Decherf, diplomate et auteur français, que la loi américaine sur la liberté de culte s'éloigne du corpus international ${ }^{16}$ dont elle dit tirer sa légitimité puisqu'elle isole un article unique, celui sur la liberté religieuse (7). L'article 18 de la Déclaration Universelle des droits de l'homme stipule pourtant, et dans cet ordre, que ; "Toute personne a droit à la liberté de pensée, de conscience et de religion" (ONU, 1). De fait, l'IRFA déséquilibre l'architecture de ces textes en accordant un statut privilégié à la liberté de culte alors que la liberté de conscience est conçue par la communauté séculière comme la faculté laissée à chacun d'adopter librement les doctrines religieuses, mais aussi philosophiques ou politiques qu'il juge bonnes, et d'agir en conséquence de ce choix. Le discours fondamentaliste américain, même s'il est parfois modéré, marque donc de son empreinte le contenu de chaque rapport; les chapitres ou les sujets traités étant majoritairement le reflet d'une idéologie visant à donner un seul fondement spirituel à l'organisation du corps social. Le message tel qu'il est articulé dans les rapports encourage les individus à définir leur identité non pas par l'appartenance à une culture collective et nationale, mais par une identification à une croyance religieuse. La question de l'identification confessionnelle étant particulièrement prégnante dans l'histoire contemporaine de l'Égypte, il convient à présent de s'intéresser aux réactions des opposants autochtones et aux arguments énoncés à l'encontre de cette hiérarchisation des droits.

\section{Modèle idéologique contre réalité sociale : les raisons du rejet}

L'année qui précède le vote de la loi américaine, le raïs égyptien Hosni Moubarak est reconduit dans ses fonctions après avoir obtenu $97 \%$ des voix lors de la présidentielle. Il sera ainsi réélu en 1999 et 2005. Si le verdict des urnes a été de nombreuses fois remis en question dans la rue, le régime autoritaire a maintenu l'état d'urgence et résisté jusqu'à la démission contrainte de son chef le 11 février 2011. Au cours des deux dernières décennies, le pouvoir exploite les clivages sociaux pour asseoir son autorité. Les partis politiques, autant que les communautés religieuses, rivalisent pour obtenir les faveurs du gouvernement. Telle est l'observation du sociologue Mohammed Bamyeh: «Le gouvernement était considéré de la même manière par ses élites et par ses électeurs comme une source de faveurs» $(56)^{17}$. C'est dans ce contexte que le 22 mars 2001 une délégation américaine conduite par le président de l'USCIRF, Elliott Abrams s'est rendue au Caire afin d'enquêter sur les pratiques discriminatoires. À la réception organisée par l'ambassade des États-Unis, on rapporte que seuls deux représentants de la société civile égyptienne s'y seraient rendus, l'un d'entre eux niant plus tard sa présence (Langohr, 1). En vérité, la visite de la commission a soulevé l'indignation aussi bien des coptes que des musulmans. Se faisant le relais des dignitaires offensés, la presse égyptienne pointe du doigt la duplicité des Américains qui justifient chez eux ce qu'ils condamnent chez les autres. On invoque en particulier certaines pratiques comme l'utilisation de «preuves 
secrètes » autorisée par le Secret Evidence Act de 1996 qui permettait de maintenir les accusés en détention sans les informer des raisons de leur incarcération (Abdel-Latif, 1). On dénonce aussi les écoutes téléphoniques ou les perquisitions pourtant interdites par le $4^{\text {ème }}$ amendement mais rendues possibles par le Patriot Act de 2001 (Abdel-Latif, 2). Par ailleurs, plusieurs associations égyptiennes pour la défense des droits de l'homme soulignent le double discours de l'USCIRF qui ne condamne guère la violation des droits civiques des Palestiniens dans les territoires occupés (Langohr, 2). Mais au-delà de ces critiques, somme toute prévisibles, quels sont les effets de l'action américaine sur la dynamique conflictuelle de la société égyptienne contemporaine?

15 C'est en premier lieu sur les tensions interconfessionnelles que les effets de l'action américaine sont les plus manifestes. Bien que victimes de violences répétées et de discrimination, certains coptes ont expliqué que leur communauté faisait partie intégrante de la nation égyptienne et qu'elle ne devait pas être considérée comme une minorité puisque son existence en terre du Nil est antécédente à l'arrivée des musulmans au VII ${ }^{e}$ siècle $^{18}$. Par conséquent, en désignant ainsi les coptes, les agents de l'USCIRF singularisent plus encore ces populations qui deviennent alors des boucs émissaires, coupables de trahison. L'accusation est d'autant plus répandue que le particularisme copte est cultivé par une partie de l'élite chrétienne qui soutient que cet ancrage dans le monde pharaonique exclut de fait toute affiliation au monde arabe. Voici ce que déclarait à ce sujet l'évêque du diocèse de Al-Qusiya en 2008 : «Si vous dites à un copte qu'il est arabe, vous l'offensez [...]. Nous ne sommes pas arabes, nous sommes égyptiens. Je suis très heureux d'être égyptien et je n'accepterais pas d'être un 'arabe', car d'un point de vue ethnique je n'en suis pas un » (cité dans Guirguis, 70). De plus, la prise de position du pape Chenouda et du synode des évêques en faveur de la réélection de H. Moubarak en 2005 a de la même façon fortement envenimé les relations entre chrétiens et musulmans, surtout parmi les leaders du mouvement d'opposition Kifâya créé en 2004. Indigné par cette manœuvre, l'écrivain et membre fondateur du mouvement Alaa al Aswany écrit : « Le pope Chenouda représente une autorité spirituelle et non politique. [...] Il utilise ses attributs religieux pour imposer son point de vue politique aux coptes. En faisant cela, il confisque leur droit de changer d'opinions » (157).

16 Ainsi, la singularisation des coptes a pour effet de renforcer le sentiment communautaire et par conséquent d'encourager la confessionnalisation de la vie politique. L'approche américaine serait à l'image, pour beaucoup d'Égyptiens musulmans, de la stratégie adoptée en leur temps par les puissances colonisatrices (ottomane ou européennes) qui consistait à opposer les minorités religieuses, à «diviser pour mieux régner ». En conséquence, il est possible d'imaginer que les persécutions infligées aux chrétiens en 2005, par exemple, n'ont pas été uniquement motivées par des antagonismes religieux, mais aussi peut-être par des divergences stratégiques concernant la marche à suivre pour se débarrasser du raïs. Les assaillants, mentionnés dans les rapports de l'USCIRF, ont donc, sans doute, été tout autant animés par un ressentiment de citoyen que de croyant.

17 Au bout du compte, l'idéologie véhiculée par la commission encourage la formation d'une société égyptienne composée essentiellement de communautés religieuses, excluant ou minorant de fait tout autre forme de lien social et surtout toute autre forme de mobilisation. Comment dans ce contexte parvenir à la formation de partis politiques associant musulmans et chrétiens? C'est ce que réclame depuis longtemps le Néo-Wafd, héritier du plus ancien parti laïc et libéral égyptien, et dont le mot d'ordre est: « La religion est à Dieu et la patrie est à tous » (cité dans Mazourki, 50). De la même manière le 
philosophe Murad Wahba, très critique des mouvements fondamentalistes, a déclaré dans le journal Al-Ahram: "Etre croyant ou citoyen sont deux choses différentes " (1) ${ }^{19}$, témoignant ainsi de l'existence, dans la société civile égyptienne, d'un courant musulman et séculariste opposé à toute instrumentalisation de la religion, à toute atteinte à l'identité collective et nationale.

Seulement, ce n'est pas uniquement sur les relations interconfessionnelles que semble peser la mission américaine. L'ignorance de la tradition séculière chrétienne mobilise, là aussi, de nombreux intellectuels ou leaders politiques. S'attachant à condamner la singularisation des coptes, l'historienne Vivian Ibrahim rappelle que la communauté en question est loin de former un groupe monolithique : « Dans la plupart des analyses et des imaginaires on estime a priori que les coptes agissent de manière unitaire et uniforme » (1). Il faut en effet garder en mémoire que la fracture entre coptes laïcs et religieux s'est considérablement aggravée en 1971 avec l'intronisation de Chenouda III dont la gestion des affaires communautaires a été marquée, nous l'avons évoqué, par une politisation de la fonction patriarcale. Décidé à régner sans partage, Chenouda a marginalisé les coptes non-pratiquants en reprenant, par exemple, la gestion de nombreuses associations culturelles ou caritatives initialement créées par des laïcs (Guirguis, 137). La composante séculière refuse cependant que l'Église décide seule, par exemple, de la formulation des droits de la famille, notamment en matière de divorce. Parce qu'ils étaient évincés de la scène communautaire et opposés aux prises de position politique du patriarche en faveur du régime, les coptes laïcs sont dès les premiers jours de la révolution de 2011 descendus dans la rue pour s'engager dans le processus de construction d'un État de droit. La mission américaine en Égypte telle qu'elle est menée par la commission USCIRF a donc pour autre incidence d'aviver les tensions au sein même de la communauté copte.

En fait, pour les Égyptiens laïques, qu'ils soient marxistes ou libéraux, chrétiens ou musulmans, l'espoir d'un rassemblement populaire qui culmine avec la révolution a été freiné par des tentatives de divisions communautaires ou des discours visant à dissocier les composantes de la société civile. Il en ressort donc que la raison fondamentale pour laquelle les activités de la commission américaine en Égypte ont rencontré et continuent de rencontrer une opposition tient au fait que la loi de 1998 privilégie une hiérarchisation verticale de la société plutôt qu'une hiérarchisation horizontale ou stratifiée. L'engagement américain en faveur des minorités confessionnelles porterait à croire que la foi a des vertus plus structurantes que la classe sociale ou la culture commune.

\section{Conclusion}

La vision d'une Amérique élue et servant de modèle au reste du monde est, nous l'avons évoqué en introduction, une des constantes de la politique étrangère américaine. Ainsi, au cours des années 1990, la nouvelle droite chrétienne entend propager la culture et les valeurs états-uniennes en remportant la guerre des idées; le "nouveau siècle américain ", du nom du projet rédigé par les néoconservateurs, est représenté comme le modèle le plus enviable que puissent adopter les peuples de la planète. La promulgation de l'IRFA permet donc aux chrétiens évangéliques et à leurs alliés d'associer leur agenda spirituel à des préoccupations intramondaines, stratégie nouvelle d'un courant religieux qui a toujours considéré l'Amérique comme l'agent de la volonté divine. 
21 Cela dit, il a été depuis démontré que les évangéliques, entre 1998 et 2008, n'ont exercé qu'une influence limitée sur la tenue de la politique étrangère, car, en réalité, il existe depuis toujours au sein de l'appareillage étatique une opposition à toute légitimation religieuse de l'action fédérale. C'est ce que Thomas Farr, l'un des plus ardents défenseurs de l'IRFA, appelle « la culture laïque de la diplomatie américaine » $(960)^{20}$. En fait, T. Farr est forcé de conclure en 2009 : «Le problème de la liberté religieuse demeure éloigné des impératifs de la politique étrangère. Dans la plupart des cas, aucune action n'a été entreprise à l'encontre des pays désignés » (957) ${ }^{21}$. Il a été en effet observé que, par exemple, dans le sillage du 11 septembre 2001, la diplomatie américaine a préféré taire ses incriminations que perdre de précieuses alliances dans sa conduite de la guerre contre le terrorisme ${ }^{22}$.

Il n'en demeure pas moins que l'intervention d'acteurs extérieurs sur le terrain peut parfois produire le contraire de l'effet recherché; à vouloir le salut des chrétiens d'Orient, les États-Unis contribuent indirectement à leur mise en péril. En effet, si l'école de pensée wilsonienne privilégie la promotion des vertus du modèle états-unien, l'étude de certaines stratégies exploitées à cette fin démontre que l'Amérique transplante certaines des problématiques sociales auxquelles elle est confrontée. Le modèle développé par les concepteurs de l'USCIRF n'offre pour seul prisme d'analyse que la question religieuse. L'aveuglement traditionnel des Américains (surtout s'ils sont évangéliques) aux questions de classes réduit ainsi le débat sur la justice sociale au seul paradigme communautariste et bride toute remise en cause des inégalités structurées. Enfin, il est maintenant évident que dans la première décennie du XXI ${ }^{\mathrm{e}}$ siècle, les sociétés américaine et égyptienne étaient conjointement structurées par une polarité entre la tentative des religions de conquérir la sphère publique et le refus populaire de l'assujettissement du politique au religieux.

\section{BIBLIOGRAPHIE}

Abdel-Latif, Omayma, « US Commission Faces Closed Doors » in Al-Ahram Weekly On-Line, http:// weekly.ahram.org, http://weekly.ahram.org.eg/2001/526/eg1.htm, page consultée le 12/10/14.

Al Aswany, Alaa, Chroniques de la révolution égyptienne, Paris : Actes Sud, 2011.

Auerswald, Philip and John Garofano (eds.), Clinton's Foreign Policy : A Documentary Record, New York : Kluwer Law International, 2003.

Bamyeh, Mohammed, «The Tunisian Revolution : Initial Reflections » in The Dawn of the Arab Uprisings : End of an Old Order?, Bassam Haddad, et al. (ed.), London : Pluto Press, 2012. pp. 49-58.

Ben Barka, Mokhtar, « La place et le rôle de la droite chrétienne dans l'Amérique de George W. Bush », in Vingtième Siècle, Revue d'histoire, N $^{\circ} 97$, L’Amérique de George W. Bush, Paris : Presses de Sciences PO, 2008. pp. 39-51; Les Nouveaux rédempteurs : le fondamentalisme protestant aux Etats-Unis , Paris : Les Éditions de l'Atelier, 1998. 
Brittingham, Angela, de la Cruz, G. Patricia, «The Arab Population » : 2000. U.S. Census Bureau, in Census 2000 Brief, December 2003, http://www.census.gov, http://www.census.gov/content/ dam/Census/library/publications/2003/demo/c2kbr-23.pdf, page consultée le 12/10/14.

Clinton, William, J., « Statement on Signing the International Religious Freedom Act of 1998 ", October 27, 1998, The U.S. Government Printing Office, www.gpo.gov, page consultée le 23/09/14.

Decherf, Dominique, « Les États-Unis au secours des 'droits de l'homme religieux' ", in Critique internationale 2/ 2002 ( $\left.\mathrm{n}^{\circ} 15\right)$. pp. 15-24.

Farr, Thomas F. and William Saunders, « The Bush Administration and America's International Religious Freedom Policy ", in Harvard Journal of Law and Public Policy 32.3, Cambridge (MA) : Harvard University Press, 2009. pp. 949-970.

Fath, Sébastien, Dieu bénisse l'Amérique : la religion à la Maison-Blanche, Paris : Éditions du Seuil, 2004.

Guirguis, Laure, Les coptes d'Égypte : violences communautaires et transformations politiques (2005-2012), Paris : Editions Karthala et IISM, 2012.

Gunn, Jeremy, T., « Religion After 9/11: When Our Allies Persecute », in Religion in the News. Fall 2001, vol. 4, Nº3. www.Trincoll.edu, http://www.trincoll.edu/depts/csrpl/RINVol4No3/religious \%20persecution.htm, page consultée le 05/09/14.

Hertzke, Allen D., Freeing God's Children : The Unlikely Alliance for Global Human Rights, Lanham (MD) : Rowman \& Littlefield, 2004.

Ibrahim, Vivian, «The Priest and the Sheikh : Coptic Challenges in Contemporary Egypt », in Amnesty International Middle East North Africa Regional Office, http://www.amnestymena.org, http://www.amnestymena.org/en/Magazine/Issue19/

ThePriestandtheSheikhCopticChallengesEgypt.aspx?media=print, page consultée le 07/10/14.

Keppel, Gilles, Dieu bénisse l'Amérique : la religion à la Maison-Blanche, Paris : Seuil, 2004.

Langohr, Vickie, « Frosty Reception for US Religious Freedom Commission in Egypt », in Middle East Research and Information Project, www.merip.org, http://www.merip.org/mero/mero032901, page consultée le 01/10/14.

Mazourki, Nadia, L'islam, une religion américaine?, Paris : Éditions du Seuil, 2013.

Mead, Walter Russell, Special Providence, American Foreign Policy and How It Changed the World, New York : Knopf, 2001.

Organisation des Nations Unies, « Déclaration universelle des droits de l'homme », www.un.org, http://www.un.org/fr/documents/udhr/, page consultée le 15/09/14.

Paloutzian, R. F., Richardson, J. T. and Rambo, L. R, (eds.), « Religious Conversion and Personality Change », in Journal of Personality, 67. Hoboken (NJ) : Wiley-Blackwell, 1999. pp. 1047-1079.

Shea, Nina, «Prepared Statement : Religious Persecution in the Middle East ; Faces of the Persecuted ", in Hearings Before the Subcommittee on Near Eastern and South Asian Affairs of the Committee on Foreign Relations, United States Senate, One Hundred Fifth Congress First Session. Washington : U.S. Government Printing Office, 1998, www.gpo.gov. http://www.gpo.gov/fdsys/pkg/CHRG-105shrg40890/html/CHRG-105shrg40890.htm, page consultée le 24/09/14.

United States Commission on International Religious Freedom, Annual Reports. www.uscirf.gov. http://www.uscirf.gov/reports-briefs/annual-report . www.uscirf.gov/sites/default/files/resources/stories/PDFs/annualreport2013may.pdf 
www.uscirf.gov/sites/default/files/resources/stories/PDFs/annualreport2010may.pdf www.uscirf.gov/sites/default/files/resources/stories/PDFs/annualreport2008may.pdf www.uscirf.gov/sites/default/files/resources/stories/PDFs/annualreport2006may.pdf www.uscirf.gov/sites/default/files/resources/stories/PDFs/annualreport2004may.pdf www.uscirf.gov/sites/default/files/resources/stories/PDFs/annualreport2003may.pdf www.uscirf.gov/sites/default/files/resources/stories/PDFs/annualreport2002may.pdf www.uscirf.gov/sites/default/files/resources/stories/pdf/Annual_Report/2000annualreport.pdf, pages consultées le 02/09/14.

Wahba, Murad, «Secularism is key to democracy », in Al-Ahram Weekly, http://weekly.ahram.org, http://weekly.ahram.org.eg/News/4379/-/-.aspx, page consultée le 08/10/14.

Weber, Katherine, « Freed Boston Pastor Michel Louis 'Thanks God' After Egyptian Hostage Ordeal », in The Christian Post. www.christianpost.com.

http://www.christianpost.com/news/freed-boston-pastor-michel-louis-thanks-god-afteregyptian-hostage-ordeal-78322/., page consultée le 20/08/14.

\section{NOTES}

1. Notre traduction.

2. La droite chrétienne est, comme le rappelle Mokhtar Ben Barka, très hétérogène et compte parmi ses alliés: "des protestants mainline (luthériens, presbytériens), des juifs messianiques (appelés « juifs pour Jésus ») et des catholiques conservateurs » $(1998,40)$.

3. Dès 1820, les pasteurs américains établissent des missions à Beyrouth, puis en Égypte en 1854 . Sur ce sujet voir l'ouvrage très complet de Heather Jane Sharkey, American Evangelicals in Egypt : Missionary Encounters in an Age of Empire, Princeton, PUB, 2008.

4. Dans un discours de politique étrangère, Bill Clinton déclare en 1999 : « Les États-Unis [...] ont une inévitable responsabilité dirigeante " (the unavoidable responsibility to lead). Cité dans Fath, 53.

5. Tous les rapports de la commission (2000-2013) sont accessibles sur le site Internet de l'USCIRF à l'adresse : www.uscirf.gov

6. Le pourcentage de chrétiens coptes dans la population égyptienne est difficilement quantifiable. Les sources varient de 5 à 10 millions.

7. Notre traduction.

8. Notre traduction.

9. Voir par exemple les rapports de 2002.

10. Voir l'article de Katherine Weber, «Freed Boston Pastor Michel Louis 'Thanks God' After Egyptian Hostage Ordeal».

11. Voir par exemple le rapport de 2007, pp. 183-184.

12. Notre traduction.

13. Par exemple, Richard D. Land, Nina Shea ou Michael K. Young.

14. Notre traduction.

15. Notre traduction.

16. Les commissaires indiquent dans chaque rapport qu'ils s'appuient sur un corpus législatif international composé de la Déclaration universelle des droits de l'homme de 1948, ou du Pacte international relatif aux droits civils et politiques de 1966.

17. Notre traduction.

18. Sur l'affirmation d'une spécificité culturelle copte, consulter Guirguis, pp. 65-66.

19. Notre traduction.

20. Notre traduction.

21. Notre traduction. 


\section{RÉSUMÉS}

Lorsque le président Clinton signe l'International Religious Freedom Act en 1998, il offre la possibilité aux partisans de la droite chrétienne de promouvoir pour la première fois leur vision du monde à travers la politique fédérale américaine. L'analyse des rapports publiés entre 2000 et 2013 sur la liberté religieuse en Égypte nous permettra de mettre au jour les implications idéologiques du discours adopté par les membres de la commission. Il s'agira aussi de confronter ce discours à celui des intellectuels ou dignitaires égyptiens opposés au travail des Américains ; comment comprendre ces réticences lorsque l'on est membre d'une minorité confessionnelle et que l'on subit de manière récurrente la violence de ses compatriotes affiliés à une autre communauté religieuse?

When President Clinton signed into law the International Religious Freedom Act in 1998, he provided the members of the religious right with a unique opportunity to promote their vision of the world through the publication of annual reports assessing religious freedom worldwide. This paper presents a critical analysis of the Commission's reports (2000-2013) on the situation in Egypt and discusses the ideological implications of the discourse adopted by the commissioners. In a second step, this discourse will be confronted to the arguments put forward by members of minority groups who oppose any legislation establishing the US as the religious police of the world.

\section{INDEX}

Mots-clés : liberté religieuse, fondamentalisme chrétien, politique étrangère américaine, Église copte, Égypte

Keywords : religious freedom, Christian fundamentalism, US Foreign policy, Egypt, Coptic Church

\section{AUTEUR}

\section{DOMINIQUE CADINOT}

Université d'Aix-Marseille

dominiquecadinot@yahoo.fr 